\title{
Patients with uncomplicated coronary artery disease have reduced heart rate variability mainly affecting vagal tone
}

\author{
B Wennerblom, L Lurje, H Tygesen, R Vahisalo, Å Hjalmarson
}

\begin{abstract}
Aim-To investigate whether uncomplicated chronic coronary artery disease causes changes in heart rate variability and if so, whether the heart rate variability pattern is different from that described in patients with acute myocardial infarction.

Methods-Heart rate variability was studied in 65 patients with angina who had no previous myocardial infarcts, no other diseases, and were on no drug that could influence the sinus node. Results were compared with 33 age matched healthy subjects. The diagnosis of coronary artery disease in angina patients was established by coronary angiography in 58 , by thallium scintigraphy in six, and by exercise test only in one. Patients and controls were Holter monitored 24 hours outside hospital, and heart rate variability was calculated in the frequency domain as global power (GP: $0.01-1.00 \mathrm{~Hz}$ ), low frequency peak (LF: 0.04-0.15 Hz), high frequency peak (HF: $0.15-0.40 \mathrm{~Hz}$ ), $\mathrm{LF} / \mathrm{HF}$ in $\mathrm{ms}^{2}$, and in the time domain as SDNN (SD of normal RR intervals), SDANN (SD of all five minute mean normal RR intervals), SD (mean of all five minute SDs of mean RR intervals), rMSSD (root mean square of differences of successive normal RR intervals) (all in ms), and pNN50 (proportion of adjacent normal RR intervals differing more than $50 \mathrm{~ms}$ from the preceding $\mathrm{RR}$ interval) as per cent.

Results-The mean age in patients and controls was 60.4 (range 32-81) and 59.1 (32-77) years, respectively (NS), the male/female ratio, 57/65 and 24/33 (NS), and the mean time of Holter monitoring, $23.0(18-24)$ and 22.8 (18-24) hours (NS). Mortality in angina patients was $0 \%$ $(0 / 65)$ at one year, $0 \%(0 / 56)$ at two years, and $3 \%(1 / 33)$ at three years. Compared with healthy subjects angina patients showed a reduction in GP ( $p=0.007), \mathrm{HF}(\mathrm{p}=0.02), \mathrm{LF}(\mathrm{p}=0.02)$, $\operatorname{SD}(\mathrm{p}=0.02), \mathrm{rMSSD}(\mathrm{p}=0.01)$, and $\mathrm{pNN} 50(\mathrm{p}=0.01)$. No significant difference was found in $\mathrm{RR}, \mathrm{LF} / \mathrm{HF}, \mathrm{SDNN}$, or SDANN.

Conclusions-Uncomplicated coronary artery disease without previous acute myocardial infarction was associated with reduced high and low frequency heart rate variability, including vagal tone. SDANN and SDNN, expressing ultra low and very low frequencies which are known to reflect prognosis after acute myocardial infarction, were less affected. This is in agreement with the good prognosis in uncomplicated angina in this study.

(Heart 2000;83:290-294)
\end{abstract}

Keywords: heart rate variability; angina pectoris; coronary artery disease; myocardial infarction; prognosis

Heart rate variability reflects cardiac autonomic tone $\mathrm{e}^{1-3}$ and is a powerful and independent predictor of mortality after acute myocardial infarction. ${ }^{45}$ In animal experiments, reduced vagal and increased sympathetic tone have been found to lower the threshold for ventricular fibrillation ${ }^{6-10}$ and thus it has been assumed that changes in the autonomic balance may play a causal role in mortality after acute myocardial infarction. ${ }^{10}$ Clinical investigations of survivors ${ }^{11}$ and necropsies of non-survivors ${ }^{12-14}$ of ventricular fibrillation outside hospital have shown that this arrhythmia is usually triggered by chronic coronary artery disease and not by acute myocardial infarction. The proportion of these patients with uncomplicated coronary artery disease- that is, angina patients with no signs of previous myocardial infarction-has been reported to be approximately $30-70 \% .{ }^{12-14}$ Thus, in a significant number of sudden cardiac death victims, ventricular fibrillation seems to be triggered by true uncomplicated coronary artery disease. Reduction in heart rate variability in uncomplicated coronary artery disease with previous myocardial infarction has been described by some investigators. ${ }^{15-17}$ It is not clear, however, whether this reduction reflects previous myocardial infarction or whether coronary artery disease per se can affect heart rate variability. Our aim in this study was to investigate whether uncomplicated chronic coronary artery disease causes changes in heart rate variability and if so, whether the heart rate variability pattern is different from that described in patients with acute myocardial infarction.

\section{Methods}

PATIENTS

The aim of a larger project was to investigate heart rate variability in patients with angina pectoris, both at baseline and after the implementation of pharmacological treatment (trial 1) or after percutaneous transluminal coronary angioplasty (PTCA) (trial 2). Patients with no previous myocardial infarcts, normal ejection fraction, and no other diseases or drugs that could influence the sinus node 
were included in both trials. The aim of the present study was to study the patients at baseline.

Patients in trial 1 were admitted to Sahlgrenska University Hospital, Department of Cardiology, for evaluation of angina pectoris of recent onset and were included if they were on no drugs and showed at least $0.1 \mathrm{mV}$ of horizontal or downsloping ST segment depression in at least one precordial ECG lead during exercise testing.

Patients in trial 2 were on the waiting list for PTCA for one or two vessel disease, and complete revascularisation was expected. Drugs known to affect the sinus node were discontinued four to seven days before Holter monitoring.

Thirty three healthy age matched subjects served as controls. They had no signs or symptoms of disease, were not on drug treatment, and had a normal ECG at rest and during exercise.

All patients and subjects signed an informed consent form, and the study was approved by the local ethics committee.

\section{Methods}

Holter monitoring was performed for 24 hours before any treatment was started in patients in trial 1 , and four to seven days after discontinuation of all drugs with the exception of aspirin and antihyperlipaemic agents in trial 2 . In general, monitoring of PTCA candidates was done outside hospital during the week preceding PTCA, in order to minimise the psychological stress present in the period immediately before the procedure.

All patients kept a diary in which they recorded the start and end of periods of chest pain, their intake of drugs, and periods of recumbency.

The 24 hour recordings were digitised on a Marquette series 8000 Holter scanner (Milwaukee, Wisconsin, USA) and submitted to the standard Marquette algorithms for QRS labelling and editing. The tapes were then manually edited by a Holter research technician to assure that classification of the QRS was correct. Heart rate variability was then analysed on Marquette heart rate variability software, version 002A. This program provides measurements for spectral components using the fast Fourier transform algorithm, after elimination of ectopic beats and artefacts and substitution by interpolated beats. The system presents time and frequency domain data for 24 hours and frequency domain data in one hour periods.

A 24 hour recording was accepted for analysis if at least 18 hours were readable, including at least four hours during the night (00.00-08.00). One hour was accepted if at least 45 minutes were readable and contained no more than one ectopic beat a minute on average.

Heart rate variability data in the frequency domain were analysed as global power (GP; $0.01-1.0 \mathrm{~Hz})$, power of the high frequency peak (HF; 0.15-0.40 Hz), power of the low frequency peak (LF; $0.04-0.15 \mathrm{~Hz})$, and the $\mathrm{LF} / \mathrm{HF}$ ratio. In the time domain, heart rate variability was expressed as SDNN (standard deviation of normal RR intervals), SDANN (standard deviation of all five minute mean normal RR intervals), SD (mean of all five minute standard deviations of mean RR intervals), rMSSD (root mean square of differences of successive normal RR intervals), and pNN50 (proportion of adjacent normal RR intervals differing more than $50 \mathrm{~ms}$ from the preceding $\mathrm{RR}$ interval, given as a percentage). It is accepted that vagal tone is expressed by $\mathrm{HF}, \mathrm{SD}$, rMSSD, and $\mathrm{pNN} 50$, and sympathovagal balance by $\mathrm{LF} / \mathrm{HF}$. SDANN is considered equivalent to ultra low (ULF) and very low frequencies (VLF) in the frequency domain. GP in the frequency domain on the Marquette system includes only frequencies higher than $0.01 \mathrm{~Hz}$; this means that GP mainly reflects LF and HF, VLF to only a small extent, and ULF not at all. SDNN, on the other hand, is a true measure of total variability and reflects ULF, VLF, LF, and $\mathrm{HF}$.

Ejection fraction was as a rule calculated from the left ventriculogram at coronary

Table 1 Characteristics of patients and controls

\begin{tabular}{|c|c|c|c|c|c|c|}
\hline & \multicolumn{3}{|l|}{ Angina patients } & \multirow[b]{2}{*}{ Controls } & \multirow[b]{2}{*}{$p_{1}$} & \multirow[b]{2}{*}{$p_{2}$} \\
\hline & All & Trial I & Trial II & & & \\
\hline No & 65 & 31 & 34 & 33 & & \\
\hline Age (years) & 60.4 & $59.6(46$ to 81$)$ & $60.8(32$ to 80$)$ & 59.1 (32 to 77$)$ & NS & NS \\
\hline Male/female (n) & $57 / 8$ & $27 / 4$ & $30 / 4$ & $24 / 9$ & NS & NS \\
\hline Smokers (n) & 15 & 9 & 6 & 6 & NS & NS \\
\hline Holter duration (h) (mean, range) & $23.0(18$ to 24$)$ & $22.9(19$ to 24$)$ & $23.1(18$ to 24$)$ & $22.8(18$ to 24$)$ & NS & NS \\
\hline Angina duration (months) (median, range) & & $3(0.3$ to 12$)$ & 7 (1 to 240$)$ & - & & 0.008 \\
\hline One vessel disease $(n)$ & 34 & 7 & 27 & - & & \\
\hline Two vessel disease (n) & 18 & 11 & 7 & - & & 0.0001 \\
\hline Three vessel disease (n) & 8 & 8 & 0 & - & & \\
\hline CCS FC 0 (n) & 2 & 1 & 1 & - & & NS \\
\hline CCS FC I & 10 & 3 & 7 & - & & \\
\hline CCS FC II & 19 & 10 & 9 & - & & \\
\hline CCS FC III & 22 & 13 & 9 & - & & \\
\hline CCS FC IV & 6 & 4 & 2 & - & & \\
\hline Calculated EF (n) & 37 & 17 & 20 & - & & \\
\hline Mean \% (range) & $72(54$ to 90$)$ & $72(55$ to 88$)$ & & - & & NS \\
\hline Estimated EF (n) & 28 & 16 & 12 & - & & \\
\hline$>50 \%(\mathrm{n})$ & 28 & 16 & 12 & - & & NS \\
\hline
\end{tabular}

$\mathrm{p}_{1}$, all angina patients $v$ subjects; $\mathrm{p}_{2}$, patients in trial $\mathrm{I} v$ patients in trial 2

CCS FC, angina classes established by the Canadian Cardiovascular Society; EF, left ventricular ejection fraction; Trial I, patients with recently developed angina, no other disease and on no drugs; Trial II, angina patients on the waiting list for PTCA, no other diseases, drugs withdrawn 4-7 days before Holter monitoring. 
angiography and in a few cases from echocardiographic investigation. Ejection fraction was considered normal if $\geqslant 50 \%$.

\section{STATISTICS}

For ordinal variables, we used the MannWhitney U test, and for dichotomous variables such as sex and smoking, Fisher's exact test. For correlation estimates Spearman rank order correlations were used. A probability (p) value $<0.05$ was considered significant.

\section{Results}

Sixty five patients with angina pectoris (31 in trial 1 and 34 in trial 2) and 33 healthy subjects were included in the study. The diagnosis of coronary artery disease was established by coronary angiography in 58, thallium scintigraphy in six, and exercise test only in one.

The ejection fraction was normal (>50\%) in all cases but was stated with an exact value in only 37 , and was visually estimated as normal in the other cases. The mean number of accepted hours of recording, the subjects' age, and the proportion of smokers were approximately the same in the angina patients and the controls. There was a non-significant dominance of women in the control group (table 1 ).

Comparison of all 65 angina patients and the 33 healthy controls showed globally reduced heart rate variability (GP) in the frequency domain, including reduced $\mathrm{HF}$ and $\mathrm{LF}$, but the $\mathrm{LF} / \mathrm{HF}$ ratio was not significantly different in the two groups. Both HF and LF were higher during the night than during the day. The reduction of GP, LF, and $\mathrm{HF}$ was significant both in the day and at night, except for LF during the night. Time domain variables showed a significant reduction of SD, rMSSD, and pNN50 - that is, there were signs of reduced vagal tone. Mean $\mathrm{RR}, \mathrm{SDNN}$, and SDANN were not significantly reduced (table 2). In one patient SDANN was less than $50 \mathrm{~ms}$; no patient had an SDNN value less than $50 \mathrm{~ms}$.

Comparison of patients in trial 1 with those in trial 2 showed no significant difference in the distribution of Canadian Cardiovascular Soci-

Table 2 Heart rate variability in angina patients and healthy subjects

\begin{tabular}{llll}
\hline HRV & Subjects & Patients & p Value \\
\hline GP $(24 \mathrm{~h})\left(\mathrm{ms}^{2}\right)$ & $1574(1117)$ & $1042(691)$ & 0.007 \\
GP $(08-20 \mathrm{~h})\left(\mathrm{ms}^{2}\right)$ & $1355(953)$ & $877(535)$ & 0.03 \\
GP $(00-05 \mathrm{~h})\left(\mathrm{ms}^{2}\right)$ & $2620(2648)$ & $1765(1754)$ & 0.04 \\
HF $(24 \mathrm{~h})\left(\mathrm{ms}^{2}\right)$ & $149(145)$ & $90(82)$ & 0.02 \\
HF $(08-20 \mathrm{~h})\left(\mathrm{ms}^{2}\right)$ & $103(83)$ & $73(61)$ & 0.04 \\
HF $(00-05 \mathrm{~h})\left(\mathrm{ms}^{2}\right)$ & $356(579)$ & $178(225)$ & 0.03 \\
LF $(24 \mathrm{~h})\left(\mathrm{ms}^{2}\right)$ & $705(605)$ & $445(355)$ & 0.02 \\
LF $(08-20 \mathrm{~h})\left(\mathrm{ms}^{2}\right)$ & $645(513)$ & $379(273)$ & 0.01 \\
LF $(00-05)\left(\mathrm{ms}^{2}\right)$ & $1144(1365)$ & $777(982)$ & $\mathrm{NS}$ \\
LF/HF $(24 \mathrm{~h})$ & $6.1(4.4)$ & $6.2(3.2)$ & $\mathrm{NS}$ \\
RR $(24 \mathrm{~h})\left(\mathrm{ms}^{2}\right)$ & $841(127)$ & $815(104)$ & $\mathrm{NS}$ \\
RR $(08-20 \mathrm{~h})\left(\mathrm{ms}^{2}\right)$ & $741(225)$ & $723(195)$ & $\mathrm{NS}$ \\
RR $(00-05 \mathrm{~h})\left(\mathrm{ms}^{2}\right)$ & $952(310)$ & $938(238)$ & $\mathrm{NS}$ \\
SDANN $(\mathrm{ms})$ & $136(45)$ & $125(34)$ & $\mathrm{NS}$ \\
SDNN $(\mathrm{ms})$ & $151(46)$ & $136(33)$ & $\mathrm{NS}$ \\
SD $(\mathrm{ms})$ & $56(18)$ & $49(13)$ & 0.02 \\
rMSDD (ms) & $29(11)$ & $23(9)$ & 0.01 \\
pNN50 (\%) & $8.2(6.9)$ & $4.8(4.8)$ & 0.01 \\
\hline
\end{tabular}

Values are mean $(\mathrm{SD})$

GP, global power $(0.01-1.0 \mathrm{~Hz})$; HF, power of the high frequency peak $(0.15-0.40 \mathrm{~Hz})$; LF, power of the low frequency peak $(0.04-0.15 \mathrm{~Hz})$; pNN50, proportion of adjacent normal RR intervals differing more than $50 \mathrm{~ms}$ from the preceding RR interval, stated as percentage; rMSSD, root mean square of differences of successive normal RR intervals; RR, mean RR interval; SD, mean of all five minute standard deviations of mean RR intervals; SDANN, standard deviation of all five minute mean normal RR intervals; SDNN, standard deviation of normal RR intervals. ety angina classes (CCS FC) but a shorter duration of angina and more severe coronary artery disease in patients in trial I. However, no indices relating to heart rate variability differed significantly between the two groups.

There were no significant correlations between CCS FC, number of diseased vessels, or duration of angina on the one hand, and any heart rate variability index on the other.

All cause mortality during follow up of angina patients was $0 \%(0 / 65)$ at one year, $0 \%$ $(0 / 56)$ at two years, and $3 \%(1 / 33)$ at three years.

\section{Discussion}

In this study we showed that uncomplicated coronary artery disease - that is, angina without acute or previous myocardial infarction, other diseases, or drugs that could influence heart rate variability - was associated with a reduction of heart rate variability in the higher spectrum of frequencies. The two variables that are known to be the best indicators of poor prognosis after acute myocardial infarction ${ }^{45}$ and in chronic coronary artery disease ${ }^{15}$ SDNN and SDANN, respectively-were not significantly reduced. Only one patient had a value less than $50 \mathrm{~ms}$, which has been associated with a particularly poor prognosis. ${ }^{4} 15$

We chose to make our analyses from 24 hour Holter recordings, like most other studies that have investigated heart rate variability as a factor in prognosis. ${ }^{4515}$ All Holter recordings in both patients and controls were sufficiently long to include adequate portions of both day and night, which is essential for a reliable estimation of SDANN and SDNN.

Heart rate variability in chronic coronary artery disease has been studied by other investigators. ${ }^{15-17}$ Conclusions from those studies are, however, hampered by confounding factors such as inclusion of patients with previous acute myocardial infarction, ${ }^{15-17}$ left heart failure, ${ }^{15}$ and diabetes,${ }^{15}$ patients on drugs that influence the sinus node, ${ }^{15}{ }^{17}$ Holter recording done the same day as the coronary angiography - that is, under stress, ${ }^{15}{ }^{17}$ or heart rate variability recorded with techniques that do not permit differentiation between sympathetic, vagal, and other effects. ${ }^{16}$ All such complicating factors were avoided in the present study.

Heart rate variability data in the time domain in our study show a very clear reduction in short term variability (SD, rMSSD, $\mathrm{pNN} 50$ ), thought to reflect vagal tone. Heart rate variability in the frequency domain in angina patients was reduced over the entire range covered by the Marquette system $(0.01-1.0 \mathrm{~Hz})$. The LF and $\mathrm{HF}$ peaks seemed to be reduced as a part of that general reduction and when they were normalised for GP they showed no difference from the control subjects. These results suggest that the reduction in heart rate variability in angina patients is mainly caused by changes in autonomic tone. It seems reasonable to assume that vagal tone was reduced. If $\mathrm{LF} / \mathrm{HF}$ reflects the sympathovagal balance, as has been stated, ${ }^{3}$ the preserved balance in the face of reduction in 
vagal activity could imply a concomitant reduction of sympathetic tone. A more plausible interpretation, however, is that there was a reduction in vagal activity affecting $\mathrm{HF}$ and the vagal part of $\mathrm{LF}$, leaving the $\mathrm{LF} / \mathrm{HF}$ ratio essentially unchanged. The assumption that LF contains vagal activity is supported by the finding that both $\mathrm{HF}$ and $\mathrm{LF}$ were higher during the night than during the day.

It may appear a contradiction that the reduction in global power in the frequency domain was not accompanied by a corresponding decrease in SDNN in the time domain. The explanation is that global power in the Marquette system does not include frequencies below $0.01 \mathrm{~Hz}$, which represent more than $90 \%$ of the total power. ${ }^{18}{ }^{19}$ If this part of the spectrum was less affected, it might explain why GP was significantly reduced but not SDRR. Support for this interpretation is that SDANN, which reflects this very low frequency part of the spectrum, was not significantly reduced. The mechanism responsible for ULF and VLF $(<0.04 \mathrm{~Hz})$ is unknown. Thermoregulation and the renin-angiotensin system have been proposed. ${ }^{1}$

Three major studies have shown that reduced heart rate variability is a powerful and independent predictor of poor prognosis after acute myocardial infarction ${ }^{45}$ and in ischaemic heart disease. ${ }^{15}$ In two of these studies, done after acute myocardial infarction, SDNN and the "triangular index" were used. Both these variables are expressions of total power-that is, power over the entire heart rate variability spectrum. Bigger et al reanalysed their data in the frequency domain and found that power reduction of ultra low $(<0.003 \mathrm{~Hz})$ and very low $(0.003-0.04 \mathrm{~Hz})$ frequencies correlated best with poor prognosis. ${ }^{19}$ Rich et al studied heart rate variability in patients with chronic coronary artery disease but no acute myocardial infarction. ${ }^{15}$ They used SDANN, which is a non-spectral estimate of low frequency variability, and found that low values were associated with poor prognosis. Thus it seems that low frequency variability is the most important part of the heart rate variability spectrum with respect to prognosis after acute myocardial infarction ${ }^{19}$ and in chronic coronary heart disease. ${ }^{15}$ However, in the study by Rich et al patients with myocardial infarction more than four weeks old, patients with a low ejection fraction, and patients with diabetes were included. ${ }^{15}$ It is not unlikely that these diseases influenced both heart rate variability and prognosis. In addition most patients were on drugs including digoxin, which would have made the interpretation of heart rate variability data very difficult.

In the present study, known confounding factors such as previous acute myocardial infarction, a low ejection fraction, other diseases, and drugs were excluded. A possible explanation for the difference between patients and controls in our study could be that the controls were more physically active than the patients, generating more frequency variation in heart rate. The fact that the difference was found during both the day time and the night time records, however, makes this explanation unlikely. Our finding that heart rate variability was reduced over a broad range of the spectrum but with less effect in the lower part suggests that true uncomplicated coronary artery disease is associated with a different pattern of heart rate variability reduction than is found in patients after acute myocardial infarction. Kleiger et al, studying more than 800 patients after acute myocardial infarction, found that $15 \%$ had an SDNN value of less than $50 \mathrm{~ms},{ }^{4}$ and in a study of angina patients, Rich et al found an SDANN value of less than $50 \mathrm{~ms}$ in $11 \% .{ }^{15}$ In the present study no patient had an SDNN of less than $50 \mathrm{~ms}$ and only one had an SDANN less than 50 ms. It may be speculated that uncomplicated coronary artery disease mainly affects the autonomic nervous control of the heart, while previous and acute necrosis of the myocardium causes mechanical derangement of the heart giving rise to reduction in heart rate variability in the lower part of the spectrum. Our patients had zero two year mortality, giving further support for the observations by Kleiger ${ }^{4}$ and Rich $^{15}$ that reduction in heart rate variability that does not include the low frequencies seems to carry a good prognosis.

Airaksinen et al found that patients with angina on no drug treatment, but some with prior myocardial infarction, had reduced vagal tone measured as heart rate response to deep breathing. ${ }^{16}$ These results are in accord with ours, but their method precluded any exploration of other parts of the heart rate spectrum.

Hayanao et al used spectral analysis in a heart rate variability study of angina patients with different degrees of coronary artery disease, including patients with previous myocardial infarction. ${ }^{17}$ They found impairment of vagal function which is in agreement with our findings, but they did not extend their study to the lower parts of the heart rate variability spectrum. The degree of reduction in vagal tone was correlated with the extent of coronary disease in Hayano's study, but there was also a correlation with the incidence of previous myocardial infarction and with the ejection fraction. In our present study of patients with normal ejection fractions and no previous myocardial infarction, we found no correlation between the number of diseased vessels and any heart rate variability index. Neither could we find any correlation between heart rate variability and the Canadian Cardiovascular Society angina grade or the duration of angina.

LIMITATIONS OF THE STUDY

In addition to non-spectral heart rate variability, we also analysed spectral heart rate variability over the 24 hours, which is a generally accepted method for exploring circadian variation in heart rate variability. ${ }^{20}{ }^{21}$ Analysis of heart rate variability in the frequency domain demands a stationary ECG recording, which cannot be assumed to be the case in the out-of-hospital setting during 24 hours of recording. Consequently the interpretation of the autonomic modulation of heart rate in the present study was less well 
defined. Our conclusions of reduced vagal tone and less affected low frequency modulation of heart rate are, however, mainly based on results from time domain analyses and these results are supported by the concomitant reduction of GP, LF, and HF. In our own and other investigators' experience, an approximate correspondence of $\mathrm{HF}$ in the frequency domain and SD, RMSSD, and pNN50 in the time domain, as expressions of vagal activity, has been demonstrated in 24 hour recordings. ${ }^{18} 22$

The relatively small number of patients in the present study is the probable reason for the fact that the SDNN and SDANN reduction in the angina patients was non-significant. A more specific demonstration of the degree of the reduction in the different wavelengths of heart rate variability in uncomplicated coronary disease would have demanded a study of the same magnitude as that by Kleiger et al on patients after acute myocardial infarction. ${ }^{4}$ We think, however, that our conclusion that SDNN and SDANN was less affected than vagal tone is true.

\section{CONCLUSIONS}

Our study shows that patients with uncomplicated coronary disease and no previous myocardial infarction had reduced heart rate variability, mainly affecting the high and low frequencies, reflecting vagal tone, while SDNN and SDANN, which mainly involve the very low and ultra low frequencies and are important for prognosis, were not significantly affected.

1 Akselrod S, Gordon D, Ubel FA, et al. Power spectrum analysis of heart rate fluctuation : a quantitative probe of beat-to-beat cardiovascular control. Science 1981;213:2202.

2.

Pommeranz B, Macaulay RJB, Candill MA, et al. Assessment of autonomic function in humans by heart rate spec-

3 Pagani M, Lombardi F, Guzzetti S, et al. Power spectral analysis of heart rate and artificial pressure variabilities as a marker of sympatho-vagal interaction in man and conscious dog. Circ Res 1986;59:178-93. 4 Kleiger RE, Miller JP, Bigger JT, et al, and the Multicenter
Post-infarction Research Group. Decreased heart rate variPost-infarction Research Group. Decreased heart rate variability and its association with increased mortality after

5 Malik M, Farrell T, Cripps T, et al. Heart rate variability in relation to prognosis after myocardial infarction : selection of optimal processing techniques. Eur Heart $\mathcal{f}$ 1989;10: 1060-74.

6 Skinner JE, Lie JT, Entman ML. Modification of ventricular fibrillation latency following coronary artery occlusion in the conscious pigs: the effect of psychological stress and beta adrenergic blockade. Circulation 1975;51:656-67.

7 Gillis RA, Corr PB, Pace DG, et al. Role of the nervous system in experimentally induced arrhythmias. Cardiology 1976;61:37-49.

8 Verrier RL, Lown B. Autonomic nervous system and malignant cardiac arrhythmias. In: Weiner $\mathrm{H}$, Hofer MA, Stunkard AJ, eds. Brain behaviour and bodily disease. New York: Raven Press, 1981:273-91.

9 Kolman BS, Verrier RL, Lown B. The effect of vagus nerve stimulation upon vulnerability of the canine ventricle. Role stimulation upon vulnerability of the canine ventricle. Role of sympathetic-p

10 Lown B, Verrier RL. Neural activity and ventricular fibrillation. N Engl f Med 1976;294:1165-70.

11 Cobb LA, Werner JA, Trobaugh GB. Sudden cardiac death. I. A decade's experience with out-of-hospital resuscitation. Mod Concepts Cardiovasc Dis 1980;49:31-6.

12 Perper JA, Kuller LH, Cooper M. Arteriosclerosis of coronary arteries in sudden, unexpected deaths. Circulation 1975;51/52(suppl III):III27-33.

13 Lie JT, Titus JL. Pathology of the myocardium and the conduction system in sudden coronary death. Circulation 1975;51/52(suppl III):III41-52

14 Basje WJ, Baba N, Keller MD, et al. Pathology of arteriosclerotic heart disease in sudden death. II. The significance of myocardial infarction. Circulation 1975;51/ 52(suppl III):III63-9.

15 Rich MW, Saini JS, Kleiger RE, et al. Correlation of heart rate variability with clinical and angiographic variables and rate variability with clinical and angiographic variables and 1988;62:714-17.

16 Airaksinen J, Ikäheimo $\mathrm{M}$, Linnaluoto $\mathrm{M}$, et al. Impaired vagal heart rate control in coronary artery disease. Br Heart f 1987;58:592-7.

17 Hayano J, Sakakibara Y, Yamada M, et al. Decreased magnitude of heart rate spectral components in coronary artery disease. Circulation 1990;81:1271-24.

18 Heart rate variability. Standards of measurements, physiological interpretation and clinical use. Task Force of the European Society of Cardiology and the North American Society of Pacing and Electrophysiology. Eur Heart 7 1996; 17:354-81.

19 Bigger JT, Fleiss JL, Steinman RC, et al. Frequency domain measures of heart period variability and mortality after myocardial infarction. Circulation 1992;85:164-71.

20 Kamath MV, Fallen EL. Diurnal variations of neurocardiac rhythms in acute myocardial infarction. Am f Cardiol 1991; 68:155-60.

21 Lombardi F, Sandrone G, Mortara A, et al. Circadian variation of spectral indices of heart rate variability after tion of spectral indices of heart rate variability
myocardial infarction. Am Heart 7 1992;123:1521-9.

22 Wennerblom B, Lurje L, Westberg S, et al. Effects on heart rate variability of isosorbide-5-mononitrate and metoprolol in patients with recent onset of angina pectoris. Cardiology 1998;89:87-93. 